\title{
An impaired biliary bicarbonate umbrella may be involved in dysregulated autophagy in primary biliary cholangitis
}

\author{
Motoko Sasaki $^{1} \cdot$ Yasunori Sato $^{1} \cdot$ Yasuni Nakanuma $^{2}$
}

Received: 21 August 2017 / Revised: 6 January 2018 / Accepted: 19 January 2018

(c) United States \& Canadian Academy of Pathology 2018

\begin{abstract}
Dysregulated autophagy may be a central player in trehe pathogenesis of primary biliary cholangitis (PBC) by inducing autoimmune processes via abnormal expression of mitochondrial antigens such as pyruvate dehydrogenase complex, E2 component (PDC-E2) and also by inducing cellular senescence in biliary epithelial cells (BECs) in bile duct lesions in PBC. We examined the association of an impaired "biliary bicarbonate umbrella" due to dysfunction of anion exchanger 2 (AE2) with dysregulated autophagy and cellular senescence in PBC. The expression of AE2 was examined in cultured BECs treated with bile acids such as glycochenodeoxycholic acid (GCDC) and tauro-ursodeoxycholic acid (TUDCA), various cytokines (IL-4, IL-13, IFN $\gamma$, TNF $\alpha$, TGF $\beta$ ), and serum deprivation. The effect of AE2 knockdown using siRNA on autophagy, cell surface expression of PDC-E2, and cellular senescence was also examined. The expression of AE2 and its association with autophagy-related markers and senescent markers $\mathrm{p} 16^{\mathrm{INK} 4 \mathrm{a}}$ and $\mathrm{p} 21^{\mathrm{WAF} 1 / \mathrm{Cip} 1}$ were immunohistochemically determined in livers taken from the patients with PBC $(n=50)$ and 69 control diseased and normal livers. The expression of AE2 was significantly induced in the cultured BECs shortly treated with GCDC and other stresses, whereas it was significantly decreased in senescent BECs induced by GCDC and other stresses $(p<0.05)$. Dysregulated autophagy, cell surface expression of PDC-E2, and cellular senescence were significantly increased by knockdown of AE2 $(p<0.05)$. The expression of AE2 was significantly decreased in cholangitis in PBC, compared to control livers $(p<0.05)$. The decreased expression of AE2 was correlated with dysregulated autophagy, abnormal expression of PDC-E2, and cellular senescence in bile duct lesions in PBC. In conclusion, an impaired biliary bicarbonate umbrella may be involved in the pathogenesis of PBC by inducing dysregulated autophagy.
\end{abstract}

\section{Introduction}

Primary biliary cholangitis (PBC) is an autoimmune cholestatic liver disease presenting with chronic, progressive cholestasis, biliary fibrosis, and liver failure [1-3]. PBC is characterized by a high frequency of serum antimitochondrial antibodies (AMAs) and unique cholangitis

Electronic supplementary material The online version of this article (https://doi.org/10.1038/s41374-018-0045-4) contains supplementary material, which is available to authorized users.

Motoko Sasaki

m8sasaki@med.kanazawa-u.ac.jp

1 Department of Human Pathology, Kanazawa University Graduate School of Medicine, Kanazawa 920-8640, Japan

2 Department of Pathology, Fukui Saiseikai Hospital, Fukui 9188503, Japan in small bile ducts referred as chronic non-suppurative destructive cholangitis (CNSDC) [1, 4]. Several genomewide association studies (GWAS) revealed that $\mathrm{PBC}$ is associated with specific HLA and non-HLA genes relating immune systems, such as $\mathrm{T}$ cell activation [5, 6]. However, there are still many enigmas concerning the pathogenesis of PBC. For example, how do AMAs play a role in the pathogenesis of bile duct lesions [1-4, 7-9]? We previously reported that dysregulated autophagy might be a central player in the pathogenesis of bile duct lesions in PBC by inducing autoimmune process via abnormal expression of mitochondrial antigens and also cellular senescence in biliary epithelial cells (BECs) in bile duct lesions in PBC [10-18]. However, the mechanisms of dysregulated autophagy were not explored in our previous studies.

Accumulating evidence suggests that anion exchanger 2 (AE2) plays a central role in maintaining a "biliary bicarbonate umbrella" for protecting biliary mucosa and that dysfunctional AE2 may be related to the pathogenesis of 
PBC [19-22]. An impaired "biliary bicarbonate umbrella" due to dysfunction of AE2 is supposed to be critical for the toxicity of hydrophobic bile acids, such as glycochenodeoxycholic acid (GCDC), and other stresses to BECs [19, 23]. For example, Ae2a,b-deficient mouse is reportedly PBC model mice presenting serum AMA, increase of $\operatorname{IgM}$, progressive cholestasis, and portal inflammation [22]. Upregulation of miR-506 is reportedly related to the decreased expression of AE2 in PBC [24].

We hypothesized that an impaired "biliary bicarbonate umbrella" may be related to dysregulated autophagy, followed by abnormal expression of mitochondrial antigens and cellular senescence in biliary epithelial lesions in PBC. In this study, we examined the association of the expression of AE2 and dysregulated autophagy, abnormal expression of mitochondrial antigens, and cellular senescence in $\mathrm{PBC}$.

\section{Materials and methods}

\section{Culture study}

\section{Cell culture and treatments}

Mouse intrahepatic BECs were isolated from 8-week-old female $\mathrm{BALB} / \mathrm{c}$ mice and were purified and cultured as described previously [25]. The cell density of the cells was less than $80 \%$ during experiments. In several experiments, BECs were treated with GCDC, deoxycholic acid (DCA), ursodeoxycholic acid (UDCA), tauro-UDCA, Etoposid (Sigma-Aldrich, St Louis, MO), and various cytokines (IL-4, IL-13, IFN $\gamma$, TNF $\alpha$, TGF $\beta$ ) (R\&D, Minneapolis, MN). In some experiments, BECs were treated with serum deprivation for induction of autophagy, as described previously [10].

\section{Knockdown of AE2 by small interfering RNA (siRNA)}

Validated siRNA for AE2 and negative control siRNA were purchased from Santa-Cruz biotech (Santa-Cruz, CA, USA) and QIAGEN (Hilden, Germany), respectively. One day before transfection, BECs were plated in $35-\mathrm{mm}$ dishes $\left(5 \times 10^{5}\right.$ cells $)$ or Lab-Tek chambers $\left(5 \times 10^{4}\right.$ cells/well), and then the cells were transiently transfected with either AE2 or control siRNA (100 nM) using Lipofectamine 3000 (Invitrogen, Carlsbad,
CA) according to the manufacturer's protocol. Bafilomycin A1 (Baf, $5 \mathrm{nM}$ ) was used to assess autophagy flux.

\section{Real-time quantitative reverse transcriptase-polymerase chain reaction (RT-PCR)}

Total RNA was extracted from the cells with a QIAGEN RNeasy Mini kit (QIAGEN) according to the manufacturer's protocol. After cDNA was synthesized, quantitative real-time PCR was performed to measure AE2 and $\beta$ actin mRNA according to a standard protocol using the SYBR Green PCR Master Mix (Toyobo, Tokyo, Japan). Primers used are shown in Table 1. Each experiment was performed twice in triplicate, and the mean was adopted in each experiment.

\section{Immunoblotting}

The cell lysate samples $(10 \mu \mathrm{g})$ were resolved by SDSPAGE and transferred to a nitrocellulose membrane as described previously. [26] After transfer, the membranes were processed for immunoblotting as described previously [26]. The primary antibodies used are shown in Table 2. Densitometry of the resulting bands was performed using Image-J software and normalized to the loading control.

\section{Immunofluorescence staining for cultured cells}

The BECs growing in a Lab-Tek chamber were fixed and immunostained using the primary antibodies for AE2, PDCE2, LC3, or p62 (Table 2), as described previously [10]. A similar dilution of the control mouse or rabbit Immunoglobulin $\mathrm{G}$ (IgG) (Dako) was applied instead of the primary antibody as a negative control. The cells were then incubated with Alexa-488-labeled or Alexa-592-labeled anti-mouse or rabbit IgG (Molecular probes, Eugene, OR) for $30 \mathrm{~min}$, counterstained with $10 \mu \mathrm{g} / \mathrm{ml} \mathrm{4}$, 6-diamidino-2phenylindole (DAPI) and evaluated under a conventional fluorescence microscope (Olympus, Tokyo, Japan).

\section{Assessment of cell-surface expression of mitochondrial antigen}

The cell-surface expression of PDC-E2 was detected in the absence of membrane-perforating agent. Cultured BECs

Table 1 Primers used in this study

\begin{tabular}{lll}
\hline Gene & Forward & Reverse \\
\hline AE2 & 5'-GCATAAGCCCCATGAGGTGT & 5'-CCTCGAATTTTATCCAGCGG \\
$\beta$-actin & 5'-CCACCGATCCACACAGAGTA & 5'-GGCTCCTAGCACCATGAAGA \\
\hline
\end{tabular}

$A E 2$ anion exchanger 2 
Table 2 Primary antibodies used in this study

\begin{tabular}{lllll}
\hline Primary antibody & Type (clone) & Pre-treatment (FFPE) & Dilution & Source \\
\hline Immunohistochemistry & & & & \\
AE2 & Rabbit poly & eARI-BA (121 $\left.{ }^{\circ} \mathrm{C}, 5 \mathrm{~min}\right)$ & $1: 200$ & Bethyl, Montgomery, TX \\
PDC-E2 & Rabbit poly & Proteinase K (RT, 10 min) & $1: 200$ & Santa-Cruz, Santa-Cruz, CA \\
LC3 & Mouse mono (5F10) & eARI-BA $\left(121^{\circ} \mathrm{C}, 5 \mathrm{~min}\right)$ & $1: 50$ & NanoTools, San Diego, CA \\
p62 & Rabbit poly & eARI-BA $\left(121^{\circ} \mathrm{C}, 5 \mathrm{~min}\right)$ & $1: 1000$ & MBL, Nagoya, Japan \\
p16 ${ }^{\text {INK4a }}$ & Mouse mono (JC8) & eARI-BA $\left(121^{\circ} \mathrm{C}, 5 \mathrm{~min}\right)$ & $1: 100$ & Neomarkers, Freemont, CA \\
p21 ${ }^{\text {WAF1/Cip1 }}$ & Mouse mono (70) & eARI-BA $\left(121^{\circ} \mathrm{C}, 5 \mathrm{~min}\right)$ & $1: 100$ & BD Transduction, San Jose, CA \\
Immunoblotting & & & & \\
AE2 & Goat poly & - & $1: 200$ & Santa-Cruz, Santa-Cruz, CA \\
LC3 & Rabbit poly & - & $1: 200$ & MBL, Nagoya, Japan \\
p62 & Rabbit poly & - & $1: 500$ & MBL, Nagoya, Japan \\
$\alpha$-tubulin & Mouse mono (TU-01) & - & $1: 500$ & Thermo Fisher, IL \\
\hline
\end{tabular}

$A E 2$ anion exchanger 2, $P D C$-E2 pyruvate dehydrogenase complex-E2 component, $p 62$ p62/sequestosome-1, LC3 microtubule-associated proteins-light chain $3 \beta, R T$ room temperature, FFPE formalin-fixed, paraffin-embedded, eARI electronic antigen retrieval instrument (pascal, Dako), $B A 0.05 \mathrm{M}$ boric acid buffer ( $\mathrm{pH} 8)$

treated with AE2 siRNA or control siRNA were fixed in buffered $10 \%$ formalin, then immunostained with antiPDC-E2, as described previously [10]. The level of cell surface mitochondrial antigen expression was evaluated semiquantitatively by Image $\mathbf{J}$ software.

\section{Assay for cellular senescence}

SA- $\beta$-gal activity was detected by using the senescence detection kit (Bio Vision, Mountain View, CA) according to manufacturer's protocol [27]. The proportion of senescent cells in each condition was assessed at Day 4 by counting the percentage of SA- $\beta$-gal-positive cells in at least $1 \times 10^{3}$ total cells using light microscopy. In addition, cellular senescence was assessed by SenTraGor ${ }^{\mathrm{TM}}$ system (Arriani pharmaceuticals, Attica, Greece) according to manufacturer's protocol [28]. This is a sensitive method for identification of senescent cells using an analog of Sudan Black B histochemical dye coupled with biotin (GL13) [28].

\section{Human study}

\section{Classification of intrahepatic biliary tree}

The intrahepatic biliary tree is classified into intrahepatic large and small bile ducts (septal and interlobular bile ducts) by their size and distributions in the portal tracts [29]. Bile ductules, which are characterized by tubular or glandular structures with a poorly defined lumen and located at the periphery of the portal tracts $[29,30]$, are not included in the small bile ducts.

\section{Liver tissue preparation}

A total of 119 liver tissue specimens (all were biopsied or surgically resected) were collected from the liver disease file of our laboratory and affiliated hospitals. The Ethics Committee of Kanazawa University approved this study. The liver specimens in this study were $50 \mathrm{PBC}, 8$ primary sclerosing cholangitis (PSC), 38 chronic viral hepatitis $(\mathrm{CVH}), 10$ livers with extrahepatic biliary obstruction (EBO), and 13 "histologically normal" livers. All PBC were from patients fulfilling the clinical, serological, and histological characteristics consistent with the diagnosis of PBC [1]. PBC livers were staged histologically [1], and 25 and 15 of PBC were stages 1, 2 (early PBC) and stages 3, 4 (advanced PBC), respectively. Fifty PBC livers were divided into $38 \mathrm{CA} 3$ (presence of CNSDC) and 12 CA0-2 (absence of CNSDC), respectively, according to Nakanuma classification [31]. Nineteen and $19 \mathrm{CVH}$ were regarded as F0-2 and as F3, 4, respectively [32]. Eight and 30 of $\mathrm{CVH}$ cases were serologically positive for hepatitis B surface antigen and anti-hepatitis $\mathrm{C}$ viral antibody, respectively. Causes of EBO were obstruction of the bile duct at the hepatic hilum or the extrahepatic bile ducts, because of stone or carcinoma, and the duration of jaundice was less than 1 month. "Histologically normal" livers were obtained from surgically resected livers for traumatic hepatic rupture or metastatic liver tumor. Normal liver tissues were obtained from an area apart from the tumor, and carcinoma tissues were not evaluated. Chemotherapy was not performed before liver resection in all patients with histologically normal liver. 
Fig.1 Decreased expression of AE2 in senescent BECs. a Cellular senescence was assessed by senescenceassociated $\beta$-galactosidase activity (SA- $\beta$-gal) at day 4 after treatment with Etoposid (Etop, $100 \mu \mathrm{M})$, serum deprivation (Dep), GCDC $(0.5 \mu \mathrm{M})$, or DCA $(25 \mu \mathrm{M}) ; n=10$ for each group, $* p<0.05$ compared to control (Cont). b The expression of AE2 mRNA was significantly decreased in senescent BECs induced by GCDC and other stresses $(p<0.05) ;{ }^{*} p<0.05$ compared to control. c The expression of AE2 protein was significantly decreased in senescent BECs induced by GCDC and other stresses $(p<$ $0.05) ; * p<0.05$ compared to control
A
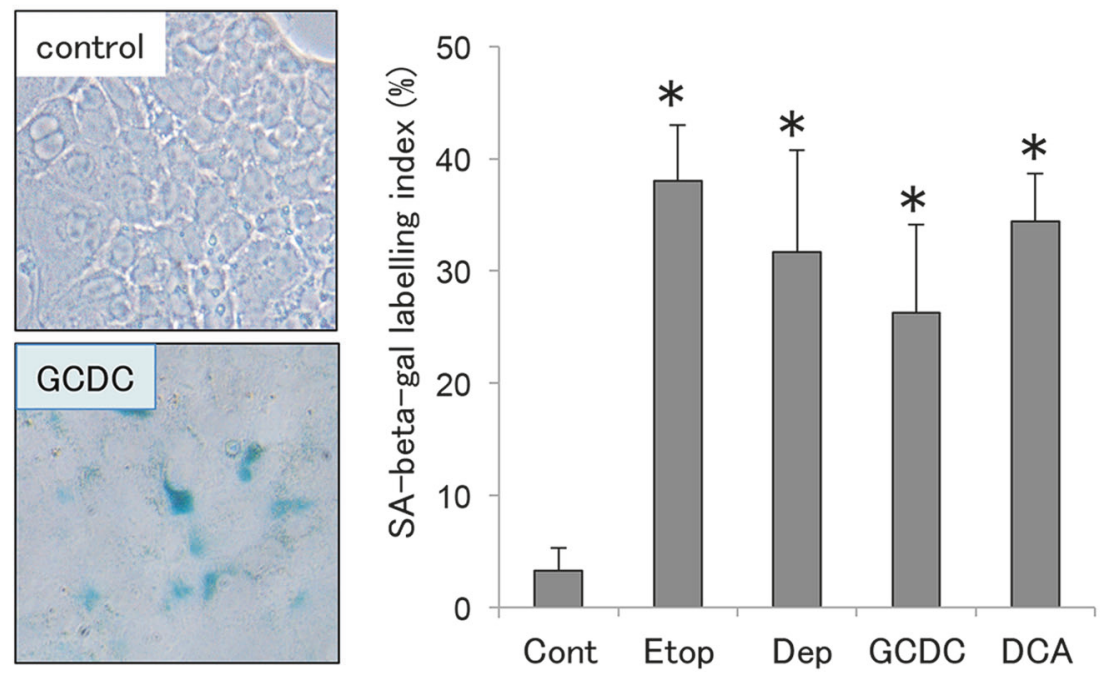

B

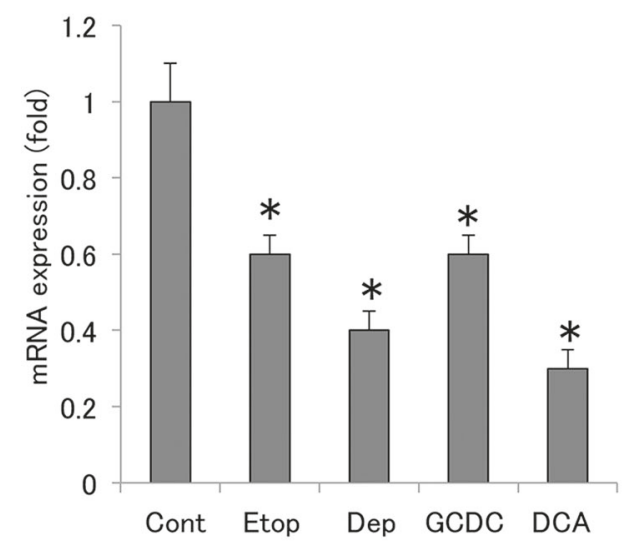

C

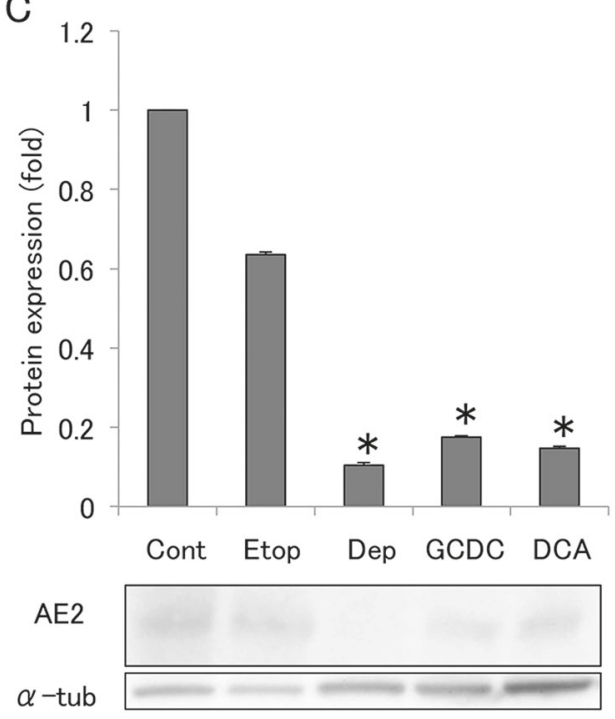

Liver tissue samples were fixed in $10 \%$ neutral-buffered formalin, and embedded in paraffin. More than 20 serial sections, $4 \mu \mathrm{m}$ thick, were cut from each block. Several sections were processed routinely for histologic study, and the remainder was processed for the subsequent immunohistochemistry.

\section{Immunohistochemistry}

We examined immunohistochemically the expression of AE2 using the primary antibody shown in Table 2, as described previously [14]. A similar dilution of the control mouse or rabbit $\operatorname{IgG}$ (Dako) was applied instead of the primary antibody as a negative control. Positive and negative controls were routinely included. Histological analysis was performed in a blinded manner. For the assessment of AE2 expression in small bile ducts, the extent of expression was evaluated as increase, no change, and decrease, compared with small bile ducts in normal liver.

\section{Double immunofluorescence}

We also performed double immunofluorescence for AE2 with autophagy related markers (LC3 and p62), senescent markers $\left(\mathrm{p} 16^{\mathrm{INK} 4 \mathrm{a}}\right.$ and $\mathrm{p} 21^{\mathrm{WAF} 1 / \mathrm{Cip} 1}$ ), and mitochondrial antigen PDC-E2. Antibodies used are shown in Table 2. In brief, either LC3, p62, PDC-E2, p16 ${ }^{\mathrm{INK} 4 \mathrm{a}}$, or $\mathrm{p} 21^{\mathrm{WAF} 1 / \mathrm{Cip} 1}$ was detected using Vector Red Alkaline Phosphatase Substrate Kit (Vector Lab, Burlingame, CA), followed by second staining for AE2 using Alexa-488-labeled antirabbit IgG. The sections were counterstained with DAPI and evaluated under a conventional fluorescence microscope. 

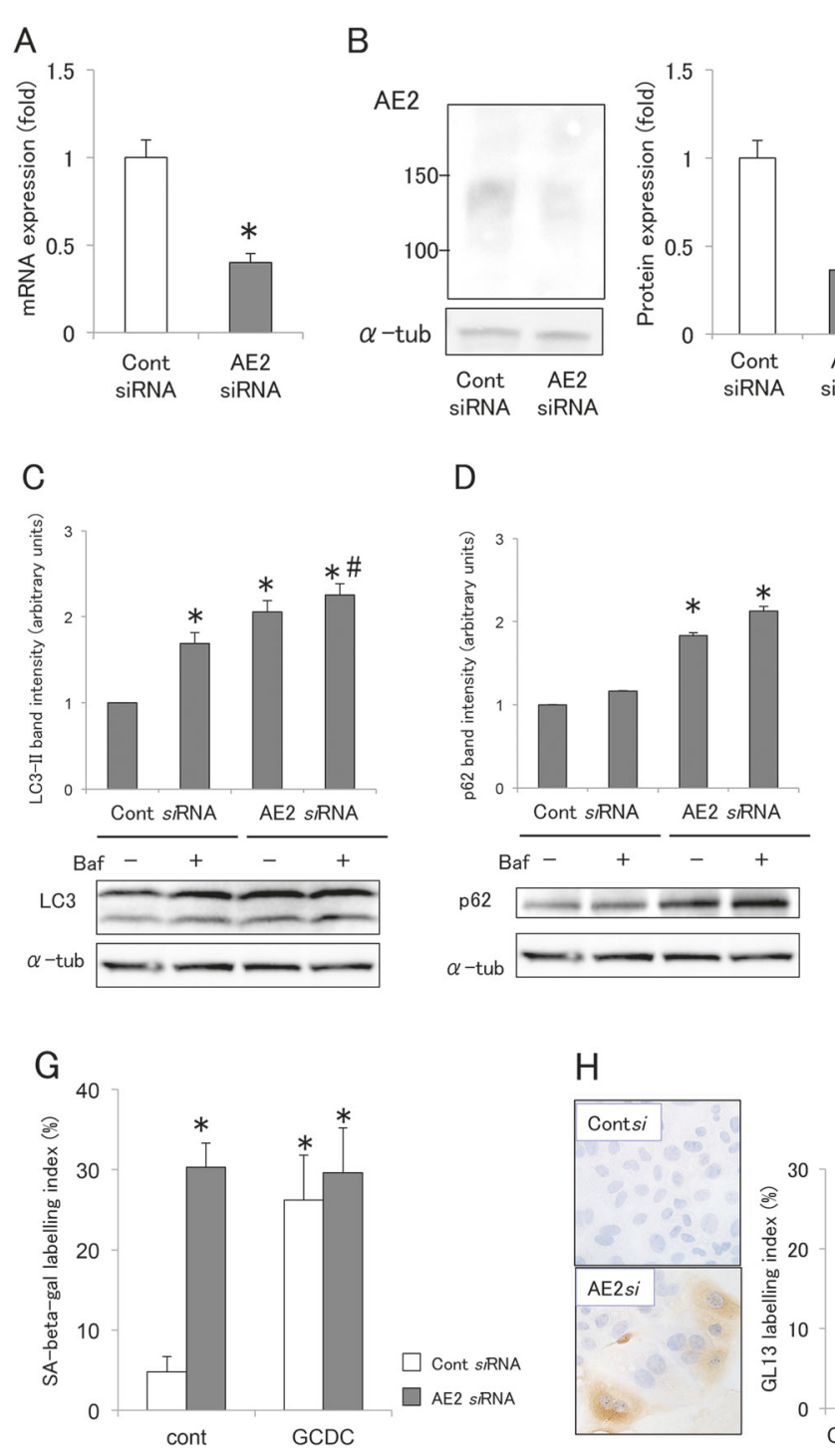
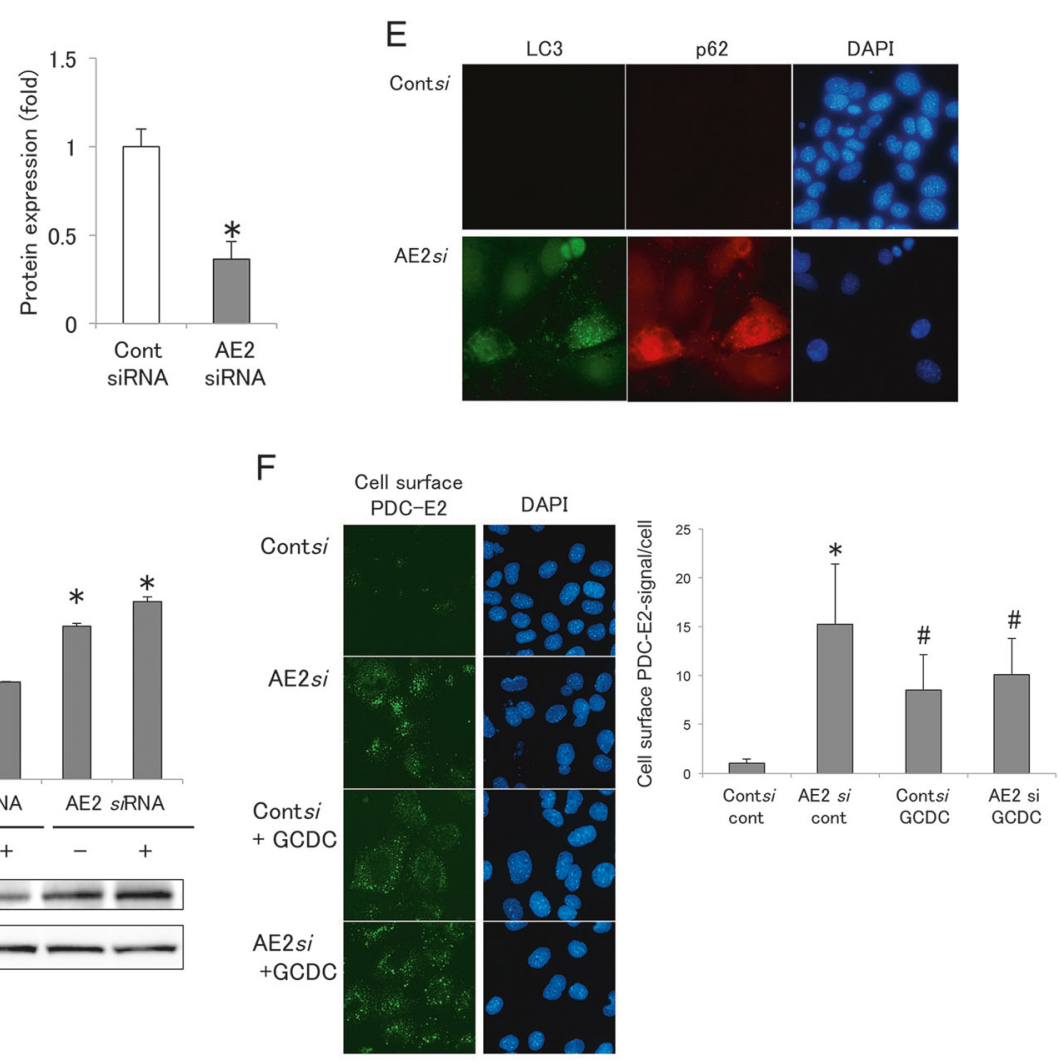

\section{Statistical analysis}

Statistical analysis of differences was performed using the Kruskal-Wallis test with Dunn's posttest. When the number of groups is 2, statistical analysis of difference was performed using the Mann-Whitney test. When the $p$ value was less than 0.05 , the difference was regarded as significant.

\section{Results}

\section{Culture study}

Increased expression of AE2 mRNA by GCDC, TUDCA, and cytokines in BECs

We examined the mRNA expression level of AE2, in cultured BECs treated with bile acids (GCDC, DCA, UDCA, tauroUDCA) and various cytokines (IL-4, IL-13, IFN $\gamma, \mathrm{TNF} \alpha$,

TGF $\beta$ ) for $4 \mathrm{~h}$. Supplemental-Fig. 1 summarizes the data. The expression of AE2 was significantly increased in cultured BECs treated with GCDC, TUDCA, IL-4, and IL-13, compared with control $(p<0.05)$.

\section{Decreased expression of AE2 mRNA in senescent BECs induced by various stressors}

Cellular senescence was induced by the treatment with Etoposid, serum deprivation, GCDC, and DCA, as reported previously (Fig. 1a). The expression of AE2 was significantly decreased in senescent BECs induced by GCDC and other stresses in mRNA and protein levels $(p<0.05)($ Fig. $1 \mathrm{~b}, \mathrm{c})$.

AE2 knockdown induced dysregulated autophagy, cell surface expression of PDC-E2, and cellular senescence

Effective knockdown of AE2 We examined the effect of AE2 knockdown on autophagy, cell surface expression of 
Fig.2 Induction of dysregulated autophagy, abnormal cell surface expression of mitochondrial antigen PDC-E2 and cellular senescence by AE2 knockdown. a The expression of AE2 mRNA in BECs treated with AE2 small interfering RNA (siRNA) or control siRNA for 1 day. AE2 mRNA was quantified with real-time PCR and normalized as a ratio using b-actin as the housekeeping gene. Data are expressed as the means $\pm \mathrm{SD} ; * p<0.01$ compared to the control, $n=3$ for each group. b The protein level expression of AE2 assessed by immunoblotting in BECs treated with AE2 siRNA orcontrol siRNA for 2 days; $* p<0.05$ vs. control $s i$ RNA, $n=3$ for each group. $\mathbf{c}$ Expression of autophagy marker, LC3-II, was greater in BECs treated with AE2 siRNA compared to BECs with control siRNA by representative western blots and quantitative analysis of LC3-II band intensity. The degree of increase in LC3-II level by treatment with Bafilomycin A1 (100 nM) was less in BECs with AE2 siRNA compared to BECs with control siRNA, demonstrating decreased autophagic flux; $* p<0.05$ compared to control siRNA, ${ }^{\#} p<0.05$ compared to AE2 siRNA. d Expression of p62 was greater in BECs treated with AE2 siRNA compared to BECs with control siRNA by representative western blots and quantitative analysis of LC3-II band intensity; $* p<0.05$ compared to control siRNA. e Double Immunofluorescent staining for LC3 and p62 in BECs treated with AE2 siRNA (AE2si) or control siRNA (Contsi) for 2 days. The expression of LC3 and p62 was absent in BECs treated with control siRNA, whereas a granular expression of LC3 and p62 was increased in the cytoplasm of BECs treated by AE2 siRNA. f Cellsurface expression of PDC-E2 in BECs treated with AE2 siRNA (AE2si) or control siRNA (Contsi) with or without glycochenodeoxycholate (GCDC, $0.5 \mu \mathrm{M}$ ) for 2 days. Left, the expression of PDC-E2 was absent on the surface of BECs treated with control siRNA, whereas the cell-surface expression of PDC-E2 was increased on the surface of BECs treated with AE2 siRNA. Immunofluorescent staining for PDC-E2 in the absence of a membrane-permeabilizing agent. Original magnification, $\times 400$. Right, semiquantitative analysis of cellsurface expression of PDC-E2 in BECs. The cell-surface signal for PDC-E2 was significantly increased in cells treated with AE2 siRNA $(15.3 \pm 6.1)$, AE2 siRNA + GCDC $(10.1 \pm 3.7)$, and control siRNA + GCDC $(8.5 \pm 3.6)$, when compared with control siRNA BECs $(1.0 \pm$ $0.4)$. Data are expressed as the mean $\pm \operatorname{SD}(n=5) ; * p<0.01$ and ${ }^{\#} p<$ 0.05 , compared to the control siRNA. g Cellular senescence in BECs treated with AE2 siRNA (AE2si) or control siRNA (Contsi) with or without GCDC for 4 days. Cellular senescence was assessed by senescence-associated $\beta$-galactosidase activity (SA- $\beta$-gal). Percentage of cells positive for SA- $\beta$-gal was significantly increased in cells treated with AE2 siRNA (SA- $\beta$-gal labeling index, 30.3 \pm 5.6 ), compared to control siRNA $(4.8 \pm 1.9)$ and treatment with GCDC tended to further accelerate cellular senescence (control siRNA + GCDC, 26.2 \pm 3.0 ; AE2 siRNA + GCDC, 29.6 \pm 5.6 ). Data were expressed as mean $\pm \mathrm{SD} ; * p<0.05$ compared to control. h Cellular senescence was assessed by SenTraGor ${ }^{\mathrm{TM}}$ system in BECs treated with AE2 siRNA (AE2si) or control siRNA (Contsi) for 4 days. Percentage of cells positive for GL13 was significantly increased in cells treated with AE2 siRNA (GL13 labeling index, 18.7 \pm 6.4 ), compared to control siRNA $(4.4 \pm 2.8)$. Data was expressed as mean $\pm \mathrm{SD} ; * p<0.01$ compared to control

PDC-E2, and cellular senescence using AE2 siRNA. An effective knockdown of AE2 using siRNA was confirmed in mRNA and protein levels (Fig. 2a, b)

AE2 knockdown induced dysregulated autophagy Immunoblotting showed that autophagy was dysregulated in BECs treated with AE2 siRNA, compared to control
siRNA. Expression of the autophagy marker, LC3-II, in BECs with AE2 siRNA was greater than in BECs with control siRNA (Fig. 2c). The expression of LC3-II was further increased in the presence of Bafilomycin A1 in both BECs with AE2 siRNA and control siRNA (Fig. 2c). The degree of increase was less in BECs with control siRNA, compared to BECs with AE2 siRNA, indicating decreased autophagic flux by AE2 knockdown (Fig. 2c). The expression of autophagyrelated p62 was increased in BECs with AE2 siRNA, compared to control siRNA, indicating dysregulated autophagy (Fig. 2d).

Immunofluorescent staining disclosed that a granular expression of LC3 and p62 was observed as dots in BECs treated with AE2 siRNA, which suggests dysregulated autophagy (Fig. 3e).

AE2 knockdown increased a cell-surface expression of PDCE2 The expression of mitochondrial antigen PDC-E2 was absent on the surface of control BECs (Fig. 2f), whereas, the cell-surface expression of PDC-E2 was increased on the surface of BECs treated with AE2 siRNA (Fig. 2f). Semiquantitative analysis disclosed that the cell-surface expression of PDC-E2 was significantly greater in BECs by knockdown of AE2 using siRNA than BECs with control siRNA $(p<0.01) \quad$ (Fig. 2f). The cell-surface expression of PDC-E2 was significantly increased by the treatment with GCDC $(p<0.05)$ (Fig. 2f), as reported previously [12]. These findings suggest that dysregulated autophagy caused by AE2siRNA may induce the increased expression of mitochondrial antigen PDC-E2 on the cell surface of BECs

AE2 knockdown induced cellular senescence Cellular senescence assessed by SA- $\beta$-gal activity was significantly increased by the knockdown of AE2 using siRNA even in control culture condition $(p<0.01)$ (Fig. 2g). The treatment with GCDC tended to further accelerate cellular senescence, although there was no significant difference. Cellular senescence assessed by SenTraGor ${ }^{\mathrm{TM}}$ system was also significantly increased by knockdown of AE2 using siRNA even in control culture condition $(p<0.01)$ (Fig. 2h).

\section{Human studies}

\section{Decreased expression of AE2 in damaged BECs in small bile ducts in PBC}

AE2 was mainly expressed in the basolateral side in BECs in small bile ducts in normal liver (Fig. 3a). Luminal expression of AE2 was increased in small bile ducts in some patients with CVH. In contrast, 
Fig.3 Expression of AE2 in small bile ducts in primary biliary cholangitis (PBC) and control livers. a Top left, AE2 was mainly expressed in the basolateral side in BECs in small bile ducts (arrow) in normal liver. Top right, Luminal expression of AE2 was increased in small bile ducts in $\mathrm{CVH}$ (arrow). Bottom, the expression of AE2 was decreased in the damaged small bile ducts (arrow) in PBC.

Whereas, it was increased in some bile ductules (thin long arrow). PBC cases \#1 and \#3 (stage 2). Immunostaining for AE2. Original magnification, $\times 400$. b Evaluation of the expression of AE2 in small bile ducts in PBC and control livers. The expression of AE2 was significantly decreased in small bile ducts in PBC with CNSDC (PBC, CA3), compared with other groups $(p<0.01)$. In contrast, the expression of AE2 was significantly increased in small bile ducts in chronic viral hepatitis $(\mathrm{CVH})$, compared with other groups $(p<0.01)$. Gray column, decrease; white column, no change; and stripe column, increase. Decrease: $* p<0.01$ compared to other groups; Increase ${ }^{\#} p<0.01$ compared to PBC, EBO, and NL. NL normal liver, PSC primary sclerosing cholangitis
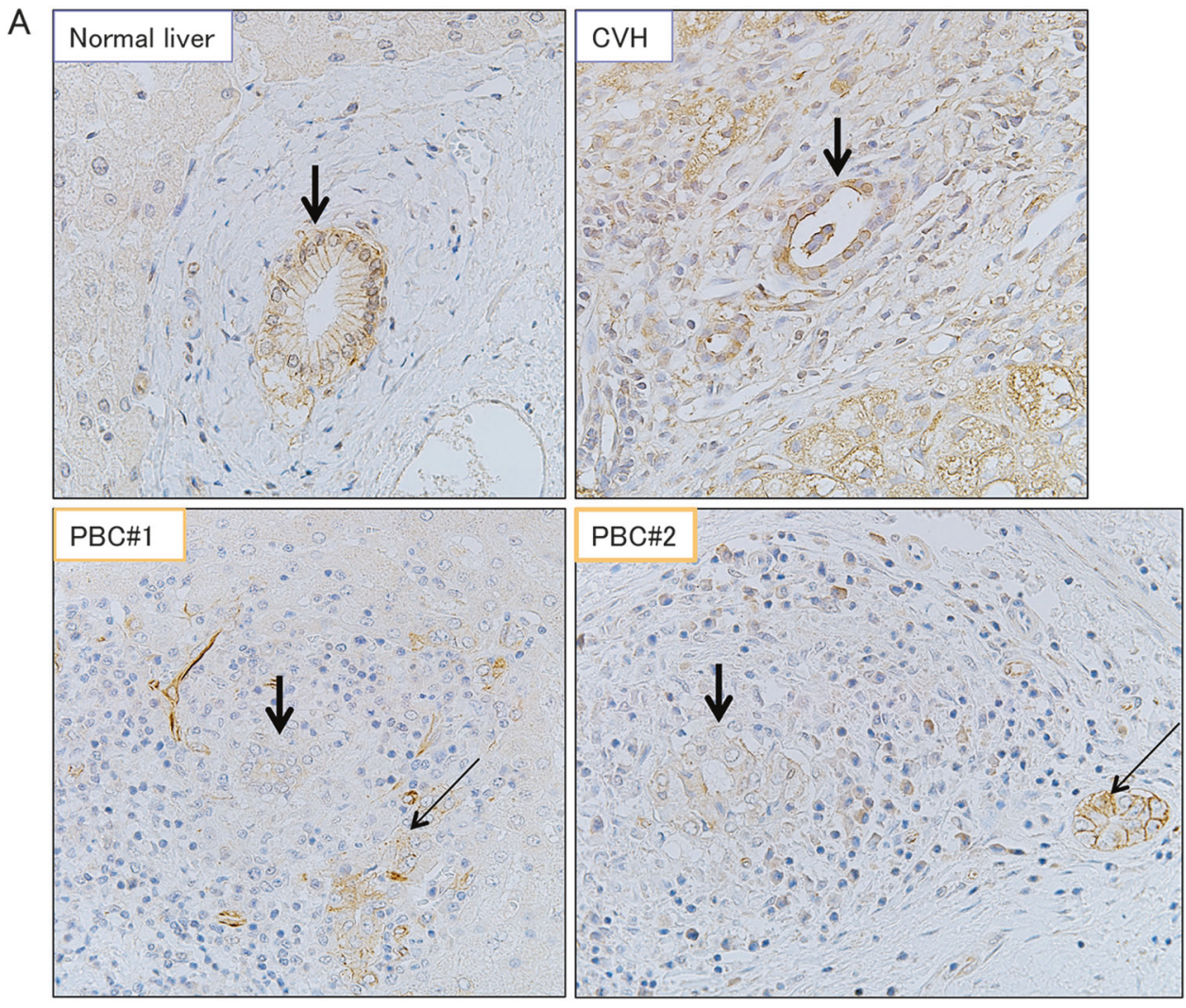

B

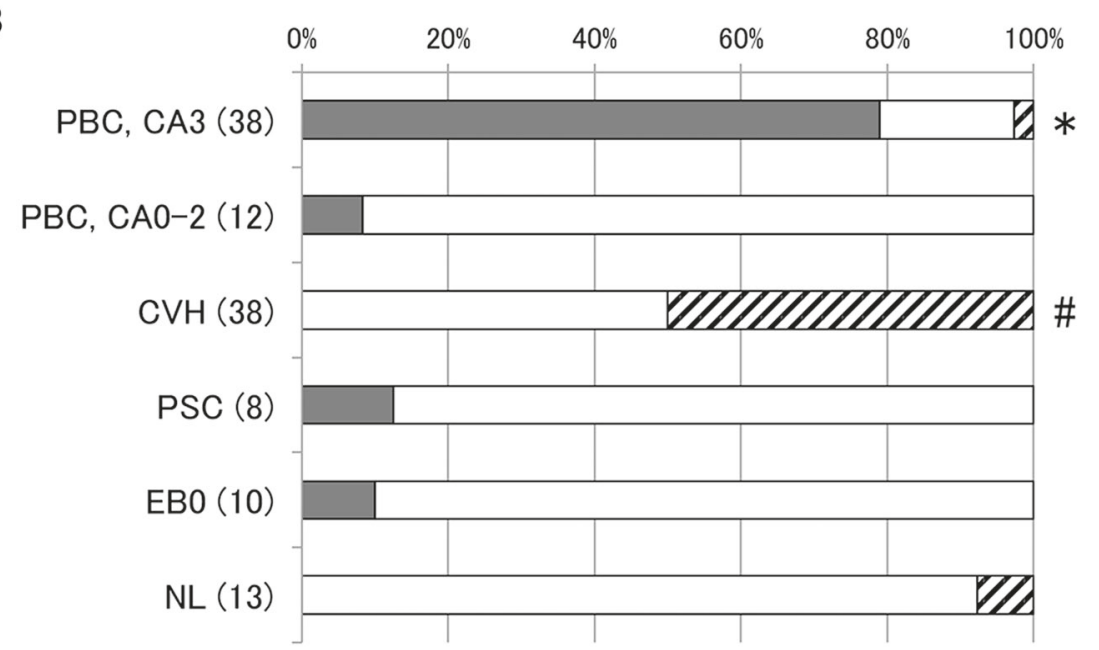

the expression of AE2 was decreased in the damaged small bile ducts (Fig. 3a). AE2 expression was increased in non-inflamed bile ducts and bile ductules (Fig. 3a).

The expression of AE2 was significantly decreased in PBC with CNSDC (PBC, CA3), compared with PBC without CNSDC (PBC, CA0-2), CVH, PSC, EBO, and normal livers $(p<0.05)$ (Fig. 3b). The expression of AE2 was significantly increased in small bile ducts in $\mathrm{CVH}$, compared with $\mathrm{PBC}, \mathrm{EBO}$, and normal livers $(p<0.01)$ (Fig. 3b).
Decreased expression of AE2 correlated with dysregulated autophagy, increased expression of mitochondrial antigen PDC-E2, and cellular senescence

Double immunostaining revealed that the expression of AE2 was frequently decreased in the damaged bile ducts in which autophagy markers (LC3 and p62), PDC-E2, senescent markers ( $\mathrm{p} 16^{\mathrm{INK} 4 \mathrm{a}}$ and $\left.\mathrm{p} 21^{\mathrm{WAF} 1 / \mathrm{Cip} 1}\right)$ were expressed in PBC (Fig. 4, Supplemental Fig. 2). Whereas, the expression of AE2 was seen in small bile ducts in control livers in which autophagy markers (LC3 and p62), PDC-E2, 

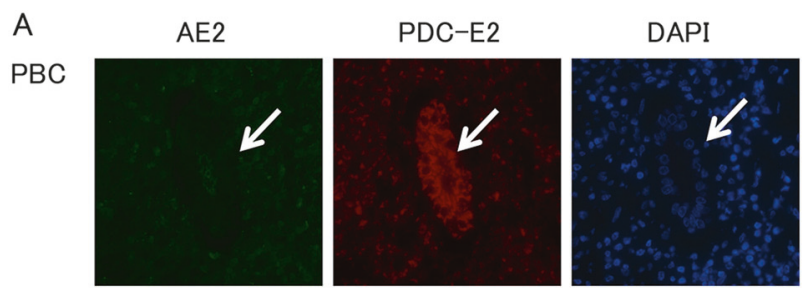

$\mathrm{CVH}$
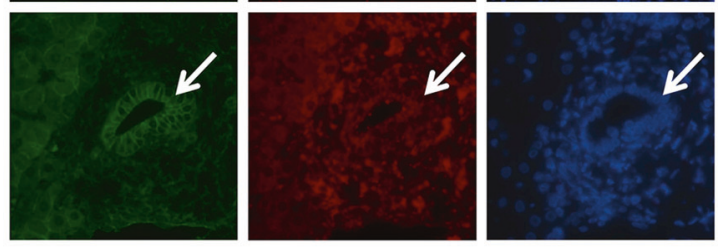

B
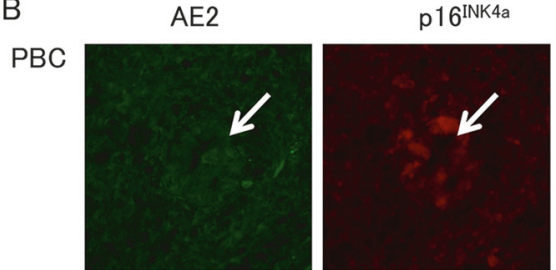

$\mathrm{CVH}$
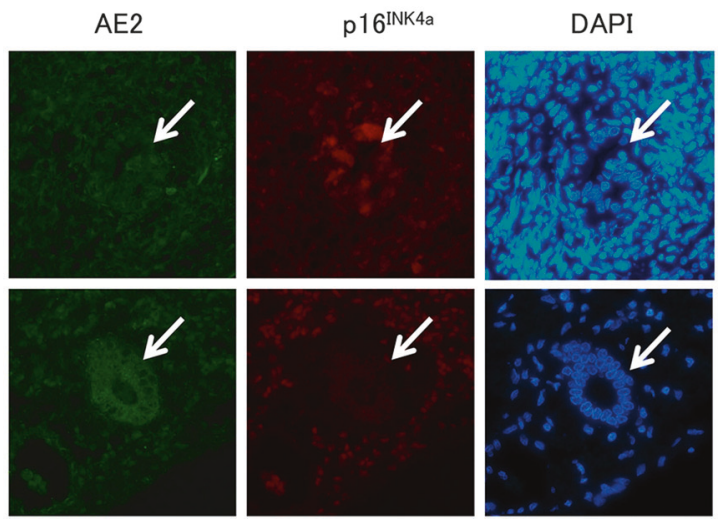

Fig. 4 Association of AE2 expression with PDC-E2 or senescent markers p16INK4a in small bile ducts in primary biliary cholangitis (PBC) and chronic viral hepatitis $(\mathrm{CVH})$. a Increased expression of PDC-E2 (top middle) was seen in a damaged small bile duct (arrow) showing decreased expression of AE2 (top left) in PBC. In contrast, the expression of PDC-E2 (bottom middle) was not seen in a small bile duct (arrow) with AE2 expression in $\mathrm{CVH}$ (bottom left). b Increased expression of p16INK4a (top middle) was seen in the nuclei of BECs in a damaged small bile duct (arrow) showing decreased expression of AE2 (top left) in PBC. In contrast, the expression of p16INK4a (bottom middle) was not seen in a small bile duct (arrow) with AE2 expression in CVH (bottom left). Double immunostaining for AE2 (green) and PDC-E2 or p16INK4a (red). Original magnification, $\times 400$

senescent markers $\left(\mathrm{p} 16^{\mathrm{INK} 4 \mathrm{a}}\right.$ and $\left.\mathrm{p} 21^{\mathrm{WAF} 1 / \mathrm{Cip} 1}\right)$ were not expressed (Fig. 4, Supplemental Fig. 2).

\section{Discussion}

The data obtained in this study are summarized as follows: (1) the expression of AE2 was significantly increased in cultured BECs treated with GCDC, TUDCA, IL-4, and IL13 for $4 \mathrm{~h}$ and it was significantly decreased in senescent BECs induced by GCDC and other stresses; (2) AE2 knockdown using siRNA induced dysregulated autophagy, cell surface expression of PDC-E2, and cellular senescence in cultured BECs; (3) the expression of AE2 was significantly decreased in small bile ducts involved in CNSDC in $\mathrm{PBC}$, compared to control livers. In contrast, it was

\section{Stress}

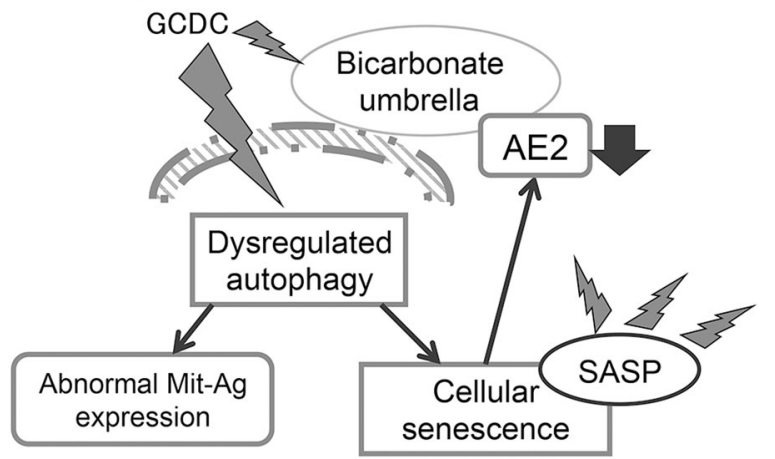

Fig. 5 Working hypothesis on the association of AE2 downregulation with dysregulated autophagy and cellular senescence in PBC. AE2 anion exchanger 2, GCDC glycochenodeoxycholic acid, Mit-Ag mitochondrial antigen, SASP senescence-associated secretory phenotype

significantly increased in small bile ducts in $\mathrm{CVH}$, compared to PBC and other livers. (4) The decreased expression of AE2 was correlated with abnormal expression of PDCE2, dysregulated autophagy, and cellular senescence in PBC.

Several lines of studies indicated that AE2, the principal bicarbonate secretor in the human biliary tree, is downregulated in PBC [19-22]. A recent study demonstrated that downregulation of AE2 may sensitize BECs to apoptotic insults activating soluble adenylyl cyclase [23]. However, the mechanisms describing how downregulated AE2 participates in the pathogenesis of PBC have not been fully clarified. In this study, we disclosed for the first time that the decreased expression of AE2 might be involved in dysregulated autophagy, followed by abnormal expression of mitochondrial antigens and cellular senescence in BECs in PBC (Fig. 5). We have previously reported that dysregulated autophagy may be a central player in the pathogenesis of PBC by inducing autoimmune process via abnormal expression of mitochondrial antigens such as PDC-E2 and also induction of cellular senescence in BECs in bile duct lesions in PBC [10-18, 33]. In fact, abnormal cell surface expression of mitochondrial antigen PDC-E2 was observed by dysregulated autophagy caused by GCDC with and without Bafilomycin A [12]. Hydrophobic bile acid such as GCDC, endoplasmic reticulum (ER) stress, and oxidative stress are candidate triggers of dysregulated autophagy; however, the exact mechanism of dysregulated autophagy was not elucidated in our previous studies [10-12, 33].

The expression of AE2 was significantly reduced in PBC, especially in the damaged small bile ducts involved in CNSDC, in agreement with previous studies [21, 34]. Furthermore, this study disclosed for the first time that the decreased expression of AE2 was closely correlated with abnormal expression of PDC-E2 and autophagy-related 
markers LC3 and p62. The present study also demonstrated that AE2 knockdown effectively induced dysregulated autophagy and cell surface expression of PDC-E2 in BECs. These findings suggest that an impaired bicarbonate umbrella caused by AE2 dysfunction may be involved in dysregulated autophagy in the pathogenesis of PBC (Fig. 5).

It is conceivable that bile acid signaling may also participate in dysregulated autophagy and other pathophysiology of BECs in PBC. Bile acid may regulate BECs through bile acid nuclear receptor, farnesoid $\mathrm{X}$ receptor (FXR), and plasma membrane receptor TGR5 [35]. FXR suppressed autophagy in hepatocytes, whereas nuclear receptor peroxisome proliferator activated receptor $\alpha$ (PPAR $\alpha)$ induced autophagy [36]. In cholestatic livers, the accumulation of bile acids caused the accumulation of autophagosomes via suppressed autophagy flux and accumulation of p62 in hepatocytes, although the signaling pathway including FXR and TGR5 was undetermined [37]. It remains to be seen how bile acids regulate autophagy and cause dysregulated autophagy and which bile acid receptors participate in the process in BECs in PBC.

Furthermore, this study demonstrates that AE2 knockdown effectively induced cellular senescence, in agreement with a recent study [34]. Our group previously reported that cellular senescence was caused via dysregulated autophagy [10]; therefore, it is likely that cellular senescence may be induced via dysregulated autophagy due to AE2 downregulation. Conversely, AE2 expression was decreased in senescent BECs induced by GCDC, serum deprivation, and other stresses. Since a short-term treatment with GCDC and other several factors rather increased AE2 mRNA expression, chronic exposure to GCDC seems to be critical to decrease AE2 expression together with the induction of cellular senescence. It is conceivable that impairment of bicarbonate umbrella, due to senescent BECs, further exacerbates injuries of BECs (Fig. 5). It has not yet been determined whether AE2 overexpression could prevent dysregulated autophagy and cellular senescence and other injuries in BECs caused by GCDC and other toxic agents.

In addition, it is now well known that senescent BECs express various chemokines and cytokines as senescenceassociated secretory phenotypes (SASPs) in PBC and PSC, which modulate inflammatory cell infiltration and fibrosis in cholangiopathies, as our group reported for the first time [17, 18, 38, 39]. Taken together, these studies indicate that it is plausible that impairment of AE2 in parallel with cellular senescence may participate in the exacerbated inflammation and other pathologic events via SASPs in PBC.

It is of interest that allelic variations in AE2 gene have a significant association with the progression rate of liver disease in PBC under UDCA therapy in a previous study [40]. Recent GWAS studies revealed several candidates of susceptible genes/loci in PBC [5, 6]; however, AE2 has not been identified as a susceptible gene, so far. Generally, most susceptible genes/loci are HLA and non-HLA genes relating adaptive and innate immunity such as cytokines, chemokines, and their receptors in $\mathrm{PBC}[5,6]$. There were few genes/loci relating to the susceptibility/vulnerability of BECs in PBC [5, 6]. Some ER stress-related genes, such as ORM1-like protein 3 (ORMDL3), which are included in susceptible genes/loci in PBC [5, 6], might be related to autophagy of epithelial cells as suggested in inflammatory bowel disease. Our previous study showed that ER stress might be involved in dysregulated autophagy and cellular senescence in BECs. Therefore, ER stress may be associated with an impairment of AE2 in BECs in PBC.

In conclusion, an impaired biliary bicarbonate umbrella may be involved in dysregulated autophagy followed by abnormal expression of PDC-E2 and cellular senescence in BECs in PBC. Therapeutic strategies to relieve the "impaired biliary bicarbonate umbrella" may be effective for PBC.

Acknowledgements This study was supported in part by a Grant-inAid for Scientific Research (C) from the Ministry of Education, Culture, Sports and Science and Technology of Japan (15K08341).

\section{Compliance with ethical standards}

Conflict of interest The authors declare that they have no conflict of interest.

\section{References}

1. Portmann B, Nakanuma Y. Diseases of the bile ducts. In: Burt A, Portmann B, Ferrell L, editors. Pathology of the liver. 6th ed. London: Churchill Livingstone; 2011. p. 491-562.

2. Kaplan MM, Gershwin ME. Primary biliary cirrhosis. N Engl J Med. 2005;353:1261-73.

3. Lindor KD, Gershwin ME, Poupon R, et al. Primary biliary cirrhosis. Hepatology. 2009;50:291-308.

4. Nakanuma Y, Ohta G. Histometric and serial section observations of the intrahepatic bile ducts in primary biliary cirrhosis. Gastroenterology. 1979;76:1326-32.

5. Nakamura M, Nishida N, Kawashima M, et al. Genome-wide association study identifies TNFSF15 and POU2AF1 as susceptibility loci for primary biliary cirrhosis in the Japanese population. Am J Hum Genet. 2012;91:721-8.

6. Mells GF, Floyd JA, Morley KI, et al. Genome-wide association study identifies 12 new susceptibility loci for primary biliary cirrhosis. Nat Genet. 2011;43:329-32.

7. Fussey SP, Guest JR, James OF, et al. Identification and analysis of the major M2 autoantigens in primary biliary cirrhosis. Proc Natl Acad Sci USA. 1988;85:8654-8.

8. Kita H, Matsumura S, He XS, et al. Quantitative and functional analysis of PDC-E2-specific autoreactive cytotoxic T lymphocytes in primary biliary cirrhosis. J Clin Invest. 2002;109:1231-40.

9. Shimoda S, Van de Water J, Ansari A, et al. Identification and precursor frequency analysis of a common $\mathrm{T}$ cell epitope motif in mitochondrial autoantigens in primary biliary cirrhosis. J Clin Invest. 1998;102:1831-40. 
10. Sasaki M, Miyakoshi M, Sato Y, et al. Autophagy mediates the process of cellular senescence characterizing bile duct damages in primary biliary cirrhosis. Lab Invest. 2010;90:835-43.

11. Sasaki M, Miyakoshi M, Sato Y, et al. A possible involvement of p62/sequestosome-1 in the process of biliary epithelial autophagy and senescence in primary biliary cirrhosis. Liver Int. 2012;32:487-99.

12. Sasaki M, Miyakoshi M, Sato Y, et al. Increased expression of mitochondrial proteins associated with autophagy in biliary epithelial lesions in primary biliary cirrhosis. Liver Int. 2013;33:312-20.

13. Sasaki M, Ikeda H, Haga $H$, et al. Frequent cellular senescence in small bile ducts in primary biliary cirrhosis: a possible role in bile duct loss. J Pathol. 2005;205:451-9.

14. Sasaki M, Ikeda H, Sato Y, et al. Decreased expression of Bmi1 is closely associated with cellular senescence in small bile ducts in primary biliary cirrhosis. Am J Pathol. 2006;169:831-45.

15. Sasaki M, Ikeda H, Yamaguchi J, et al. Telomere shortening in the damaged small bile ducts in primary biliary cirrhosis reflects ongoing cellular senescence. Hepatology. 2008;48:186-95.

16. Sasaki M, Ikeda H, Nakanuma Y. Activation of ATM signaling pathway is involved in oxidative stress-induced expression of mito-inhibitoryp21(WAF1/Cip1) in chronic non-suppurative destructive cholangitis in primary biliary cirrhosis: An immunohistochemical study. J Autoimmun. 2008;31:73-78.

17. Sasaki M, Nakanuma Y. Bile acids and deregulated cholangiocyte autophagy in primary biliary cholangitis. Dig Dis. 2017;35:210-6.

18. Sasaki M, Miyakoshi M, Sato Y, et al. Modulation of the microenvironment by senescent biliary epithelial cells may be involved in the pathogenesis of primary biliary cirrhosis. J Hepatol. 2010;53:318-25.

19. Beuers U, Hohenester S, de Buy Wenniger LJ, et al. The biliary $\mathrm{HCO}(3)(-)$ umbrella: a unifying hypothesis on pathogenetic and therapeutic aspects of fibrosing cholangiopathies. Hepatology. 2010;52:1489-96.

20. Hohenester S, Wenniger LM, Paulusma CC, et al. A biliary $\mathrm{HCO} 3$ - umbrella constitutes a protective mechanism against bile acid-induced injury in human cholangiocytes. Hepatology. 2012;55:173-83.

21. Prieto J, Qian C, Garcia N, et al. Abnormal expression of anion exchanger genes in primary biliary cirrhosis. Gastroenterology. 1993;105:572-8.

22. Salas JT, Banales JM, Sarvide S, et al. Ae2a,b-deficient mice develop antimitochondrial antibodies and other features resembling primary biliary cirrhosis. Gastroenterology. 2008;134:1482-93.

23. Chang JC, Go S, de Waart DR, et al. Soluble adenylyl cyclase regulates bile salt-induced apoptosis in human cholangiocytes. Hepatology. 2016;64:522-34.

24. Banales JM, Saez E, Uriz M, et al. Up-regulation of microRNA 506 leads to decreased $\mathrm{Cl}-/ \mathrm{HCO} 3-$ anion exchanger 2 expression in biliary epithelium of patients with primary biliary cirrhosis. Hepatology. 2012;56:687-97.

25. Katayanagi K, Kono N, Nakanuma Y. Isolation, culture and characterization of biliary epithelial cells from different anatomical levels of the intrahepatic and extrahepatic biliary tree from a mouse. Liver. 1998;18:90-98.

26. Sasaki M, Van De Water J, Kenny TP, et al. Immunoglobulin gene usage and immunohistochemical characteristics of human monoclonal antibodies to the mitochondrial autoantigens of primary biliary cirrhosis induced in the XenoMouse. Hepatology. 2001;34:631-7.

27. Dimri GP, Lee $X$, Basile G, et al. A biomarker that identifies senescent human cells in culture and in aging skin in vivo. Proc Natl Acad Sci USA. 1995;92:9363-7.

28. Evangelou K, Lougiakis N, Rizou SV, et al. Robust, universal biomarker assay to detect senescent cells in biological specimens. Aging Cell. 2017;16:192-7.

29. Nakanuma Y, Sasaki M. Expression of blood-group-related antigens in the intrahepatic biliary tree and hepatocytes in normal livers and various hepatobiliary diseases. Hepatology. 1989;10:174-8.

30. Roskams TA, Theise ND, Balabaud C, et al. Nomenclature of the finer branches of the biliary tree: canals, ductules, and ductular reactions in human livers. Hepatology. 2004;39:1739-45.

31. Nakanuma Y, Zen Y, Harada K, et al. Application of a new histological staging and grading system for primary biliary cirrhosis to liver biopsy specimens: interobserver agreement. Pathol Int. 2010;60:167-74.

32. Desmet V, Gerber M, Hoofnagle J, et al. Classification of chronic hepatitis: diagnosis, grading and staging. Hepatology. 1994;19:1513-20.

33. Sasaki M, Yoshimura-Miyakoshi M, Sato Y, et al. A possible involvement of endoplasmic reticulum stress in biliary epithelial autophagy and senescence in primary biliary cirrhosis. J Gastroenterol. 2015;50:984-95.

34. Hisamoto S, Shimoda S, Harada K, et al. Hydrophobic bile acids suppress expression of AE2 in biliary epithelial cells and induce bile duct inflammation in primary biliary cholangitis. J Autoimmun. 2016;75:150-60.

35. Marin JJ, Macias RI, Briz O, et al. Bile acids in physiology, pathology and pharmacology. Curr Drug Metab. 2015;17:4-29.

36. Preidis GA, Kim KH, Moore DD. Nutrient-sensing nuclear receptors PPARalpha and FXR control liver energy balance. J Clin Invest. 2017;127:1193-201.

37. Kim S, Han SY, Yu KS, et al. Impaired autophagy promotes bile acid-induced hepatic injury and accumulation of ubiquitinated proteins. Biochem Biophys Res Commun. 2018;495:1541-7.

38. Tabibian JH, O'Hara SP, Splinter PL, et al. Cholangiocyte senescence by way of N-ras activation is a characteristic of primary sclerosing cholangitis. Hepatology. 2014;59:2263-75.

39. Sasaki M, Miyakoshi M, Sato Y, et al. Chemokine-chemokine receptor CCL2-CCR2 and CX3CL1-CX3CR1 axis may play a role in the aggravated inflammation in primary biliary cirrhosis. Dig Dis Sci. 2014;59:358-64.

40. Poupon R, Ping C, Chretien Y, et al. Genetic factors of susceptibility and of severity in primary biliary cirrhosis. J Hepatol. 2008;49:1038-45. 